\title{
Relationship between Psychosocial Factors and Onset of Multiple Sclerosis
}

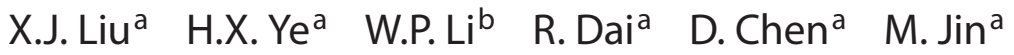 \\ a Department of Neurology, Nanfang Hospital, Southern Medical University, Guangzhou, PR China; \\ ${ }^{b}$ Department of Veterans Affairs, New Jersey Health Care System, East Orange, N.J., USA
}

\section{Key Words}

Psychosocial factors $\cdot$ Multiple sclerosis $\cdot$ Negative life events $\cdot$ Social support

\begin{abstract}
Objective: The aim of this study was to investigate the influence of psychosocial variables on patients with multiple sclerosis (MS) and the relationship between these variables and the onset of MS. Background: The current evidence indicates that many types of psychosocial factors are involved in the development and relapse of MS, and it has been suggested that they could serve as predictors as well. So far, little has been reported on the effect of psychosocial factors on MS and the relationship between psychosocial factors and the onset of MS. Methods: Forty-one patients, 15 males and 26 females, average age $37.44 \pm 12.24$ years (mean \pm $\mathrm{SD})$, were evaluated by the Life Event Scale, Eysenck Personality Questionnaire, Social Support Revaluate Scale and Symptom Check List 90 and compared with 41 equivalent healthy control subjects, 15 males and 26 females, average age $36.38 \pm 12.84$ years (mean $\pm \mathrm{SD}$ ). Disease, demographic, psychosocial and lifestyle factors were measured at baseline. Patients with MS were first diagnosed by 3 neurologists according to the Poser (1983) MS diagnostic criteria. Results: Significant differences were found between the MS and the control group in their negative emotions and symptoms such as depression, anxiety, obsession, phobia, tense inter-
\end{abstract}

personal relationship and somatization disorder. Significant differences were found between the two groups in the total number of negative life events, their family problems and the utilization of social support. The scores for various negative emotions in the MS group correlated positively with those for neuroticisms in personality type, and negatively with those for introverted and extroverted personality. Many kinds of negative emotions in the MS group correlated positively with the total number of life events, negative life events and family problems. Many kinds of negative emotions in the MS group correlated negatively with the utilization of social support. Conclusion: The psychosocial factors are closely associated with MS onset and may play important roles in the development of the disease.

Copyright $\odot 2009$ S. Karger AG, Basel

\section{Introduction}

Multiple sclerosis (MS) is an autoimmune disease characterized by demyelinating lesions originating from the white matter in the central nervous system, which often creates cognitive and emotional changes in the patients. Although the precise etiology and pathogenesis of MS are not yet known, the disease is currently thought to be the result of viral, genetic, environmental, immunological and psychosocial factors [1]. The neuropsychiatric symptoms of MS are broad and include depression, cog-

\section{KARGER}

Fax +4161306 1234

E-Mail karger@karger.ch

www.karger.com
(C) 2009 S. Karger AG, Basel

0014-3022/09/0623-0130\$26.00/0

Accessible online at:

www.karger.com/ene
Xiao-jia Liu

Department of Neurology, Nanfang Hospital

Southern Medical University

510515 Guangzhou (PR China)

Tel. +86 206164 1965, Fax +86206278 7664, E-Mail xiaojialiu@21cn.com.cn 
nitive deficits, anxiety, obsession and others [2]. Moreover, physical disability and cognitive deficits can gradually interrupt these patients' lives. The poorer quality of life may, in turn, further promote emotional and mental problems.

The role of psychosocial factors is extremely complicated in the pathogenesis of MS. Psychosocial factors may be a predictor for the onset of MS, may play an important role in the development of the disease and are closely related to the symptoms of MS. In the process of MS, the psychosocial factors and the symptoms of MS are often interrelated, and they affect both the development and outcome of the disease. As a result, they can deteriorate and ameliorate the symptoms of MS. Conversely, the symptoms can affect the psychological state of patients. For example, symptoms such as pain, fatigue, emotional changes and cognitive impairment are closely related to psychosocial factors. Recent studies show that psychosocial factors are closely related to pain, pain-related disturbances and the psychological state of patients with MS [3].

A number of studies have adopted different methods to understand the effects of psychosocial factors on the progress of MS, such as the relationship between psychological stress and the course of MS. Most of the studies show that acute short-term stress has no negative effects on the progress of MS. However, chronic psychosocial stresses, such as interpersonal conflict, loss of loved ones, lack of social support, anxiety and depression were identified as risk factors to aggravate the symptoms of patients with MS. Neuroimmunological studies have found that the lymphocytes and cytokines of patients with MS will change under different stresses, and these changes may have a clinical significance [4].

In addition, studies have shown that psychosocial factors are closely related to MS relapses, among which stress is a key factor. There have been few nonclinical factors to predict recurrence of MS (except stressful life events) in the past. Brown et al. [5] conducted a 2-year study of 101 MS patients in Australia and evaluated the patients in stressful life events, depression, anxiety and fatigue, and other conditions. The results showed that relapses were related to the frequency of acute stresses, psychological coping styles and place of birth, and unrelated to chronic stress, diseases, demographics, lifestyle, and psychosocial factors. They did not find an indirect impact on the stress-relapse relationship. Therefore, they concluded that the number rather than the severity of individual stressors was the most important risk factor to predict MS relapses, and recommended that MS patients should try to avoid situations that are likely to generate multiple stressors or which provide few avenues for social support [5].

Therefore, psychosocial factors are becoming a new area in etiology research in autoimmune diseases. We used a retrospective study to evaluate the effects of psychosocial variables on the mental health of patients with MS, as well as the relationship between psychosocial variables and the onset of MS.

\section{Methods}

\section{Participants}

This project was conducted with the full approval of the human research ethics committee at Southern Medical University. All MS patients were consecutively recruited from inpatients in Nanfang Hospital affiliated with Southern Medical University between 2000 and 2007. Patients were included in the study only if they fulfilled the criteria and had a definite diagnosis of MS according to the Poser criteria (1983). They were excluded if they were $<15$ years of age, unable to speak or read Chinese, or had a diagnosed psychotic illness (e.g. bipolar disorder), acute organic brain syndrome (e.g. delirium) or cognitive impairment that was sufficient to impair their ability to complete questionnaires and interviews (i.e. people who scored below cut-off level on three or more of the four tests of the Screening Examination for Cognitive Impairment, SEFCI) [6].

\section{Procedure}

The first step was to identify if the patients were diagnosed as having MS for the first time. After obtaining the patients' written consent, neurologists examined the participants and rated their level of physical disability; then psychologists assessed cognitive function using the SEFCI [6]. Neurological data were collected from patients prior to any discussion of stressful life events. If the participants fulfilled the included requirements, they were then asked to complete and return the study entry questionnaires 1 week after their condition became stable.

\section{Measures}

The Life Event Scale

The Life Event Scale (LES) [7] was used to calculate the total value of life events in the last 3 years before the MS diagnosis. The LES was designed by De-seng Yang and Ya-lin Zhang, based on the study of our predecessors, and was adopted in more than 10 provinces and municipalities in China since 1986 after 5 years' pre-examination. The scale is based on layered or individual points to assess mental stimulation, and includes qualitative and quantitative assessments. The 3 -year life events before the onset of MS were assessed and recorded by the interviewees themselves. The significance of this scale was explained in detail to the interviewees. The scale is a self-rating scale, containing 48 items of common life events, including three categories of issues: family life (28 questions), work and study (13 questions), social and other aspects (7 questions). Positive and negative life events scores were calculated separately. There are also two blanks attached to 
supplement the project, which are prepared for the interviewees in case they have experienced life events, but the events are not linked to the 48 items mentioned above. The evaluation standard of the scale is the higher the score, the greater the individual mental stress.

\section{Eysenck Personality Questionnaire}

The Eysenck Personality Questionnaire (EPQ) [8] was designed by the British psychologist H.J. Eysenck and others, and is an effective personality measurement tool which plays an important role in analyzing the characteristics of personality structure. In the United States, it refers to the psychological characteristics of personality that a person reflects in real attitudes and behaviors. Subsequently, the Chinese psychologist Zhong-geng Chen made an adult EPQ version based on it, which was adopted by our project. EPQ is a self-statement personality questionnaire of 88 topics, including four subscales (E, N, P, L scale) and three dimensions. E scale: 21 questions, measuring mainly introverted and extroverted personality and implicit tendency. N scale: 24 questions, measuring neuroticism or emotional stability. P scale: 23 questions, measuring psychoticism. L scale: 20 questions for the validity of the scale, assessing interviewees' camouflage. Each question in EPQ corresponds to the two options 'yes' or 'no'; interviewees may choose the answer according to their own circumstances. In our study, the original component scores were calculated and translated into standard ones in every subscale according to the appendix in EPQ.

\section{Symptom Check List 90}

The most primitive version of the Symptom Check List 90 (SCL-90) [9] was designed by L.R. Derogaitis in 1975 on the basis of his compiled Hopkin's list of symptoms (HSCL 1973). Rui-si Ge amended the described system, based on a version of the universal application which is accessible for Chinese, and developed the latest age group norm. The scale includes 90 self-assessment questions and is classified into nine factors: somatization, obsessivecompulsive symptoms, interpersonal sensitivity, depression, anxiety, hostility, horror, paranoia and psychosis. The purpose of SCL-90 is to assess whether a person has any kind of psychological symptoms and how serious they are from multiple perspectives, such as their emotions, thinking, consciousness, behavior, living habits, interpersonal relationships, food and sleep habits. It can distinguish psychological symptoms that underlie possible mental disorders.

Social Support Revaluate Scale

The Social Support Revaluate Scale (SSRS) [10] was designed by Shui-yuan Xiao in 1993 to assess individual emotional experience and satisfaction with being respected, supported and understood in society. The interviewees are required to be over 15 years old. The scale has 10 items, including three dimensions, objective support (3), subjective support (4) and social support (3). The scale has good reliability and validity, and can reflect appropriately the individual community support level. Social support has a great impact on the social life of people and can be divided into two categories: one is the objective social support, which is visible or practical, including direct material assistance, group support, participation in groups, and material support from employers, colleagues, friends, family members and relatives. The other one is subjective social support, such as individual emotional experi- ence and satisfaction in society, and is closely related to subjective feelings. The social support item is used to evaluate if interviewees refuse help from others. The higher the SSRS scores, the better the community support.

Statistical Analysis

SPSS 13.0 was used to analyze the simple t test and correlation between psychosocial factors and MS. The level of significance was set at 0.05 (two tailed). The results are expressed as p value. To avoid increasing the risk of alpha errors (false positives) we used Bonferroni corrections to adjust the $\mathrm{p}$ value. The corrected $\mathrm{p}$ value was expressed as $\mathrm{Pc}$ (P correct). The Pc value is obtained from the $\mathrm{p}$ value multiplied by the number of total factors on the scale.

\section{Results}

\section{Participants}

Out of 43 approached MS patients, 41 (95.3\%) participated in this study. Two patients did not agree to participate. The control group matched the MS group in sex, age and education level. The $41 \mathrm{MS}$ patients included 15 males and 26 females, aged 17-60 years, mean $37.44 \pm 12.24$ SD. The control group consisted of 41 healthy people, 15 males and 26 females, aged 17 to 64 years, mean $36.38 \pm$ 12.84. The education level in MS patients was 2-19 years, mean $9.09 \pm 5.37$, and in the control group it was 2.5-20 years, mean $9.89 \pm 5.53$. There were no significant differences between the two groups in age or education lev$\mathrm{el}(\mathrm{p}>0.05)$. None of the patients had any history of mental illness or cognitive dysfunction, and cooperated in inspection. The duration of MS was 3 months to 9 years, mean $2.53 \pm 1.35$ years.

\section{SCL-90 between MS and Healthy Controls}

There were significant differences in negative emotions and symptoms such as depression, anxiety, horror, obsession, tense interpersonal relationships, and somatization disorder between the MS group and the control group (table 1). There was no significant difference in hostility or paranoia between the two groups ( $p>0.05)$. The reason may be that the samples were small in number.

\section{Comparison of Psychosocial Factors between MS \\ Patients and Healthy Controls}

Significant differences were found in total number of negative life events, family problems, subjective support and utilization of social supports between the two groups. There were no significant differences in life events, positive life events, work and study problems, social and oth- 
Table 1. Results of SCL-90 of MS and healthy controls (mean \pm SD)

\begin{tabular}{llllll}
\hline Item & MS group & Healthy group & $\mathrm{t}$ & $\mathrm{p}$ & $\mathrm{Pc}$ \\
\hline Total sharing scores & $1.93 \pm 0.59$ & $1.36 \pm 0.34$ & 5.265 & $0.000^{* *}$ & $0.000^{* *}$ \\
Positive symptoms sharing scores & $2.62 \pm 0.75$ & $2.10 \pm 0.75$ & 3.132 & $0.002^{* *}$ & $0.002^{* *}$ \\
Somatization & $1.99 \pm 0.56$ & $1.37 \pm 0.45$ & 5.526 & $0.000^{* *}$ & $0.000^{* *}$ \\
Obsession & $2.05 \pm 0.69$ & $1.55 \pm 0.58$ & 3.558 & $0.001^{* *}$ & $0.009^{* *}$ \\
Interpersonal sensitivity relationship & $1.94 \pm 0.66$ & $1.33 \pm 0.38$ & 5.198 & $0.000^{* *}$ & $0.000^{* *}$ \\
Depression & $2.10 \pm 0.80$ & $1.47 \pm 0.49$ & 4.273 & $0.000^{* *}$ & $0.000^{* *}$ \\
Anxiety & $1.94 \pm 0.71$ & $1.39 \pm 0.42$ & 4.276 & $0.000^{* *}$ & $0.000^{* *}$ \\
Hostility & $1.67 \pm 0.63$ & $1.38 \pm 0.71$ & 1.936 & 0.056 & 0.504 \\
Horror & $1.77 \pm 0.78$ & $1.17 \pm 0.28$ & 4.639 & $0.000^{* *}$ & $0.000^{* *}$ \\
Paranoia & $1.67 \pm 0.72$ & $1.39 \pm 0.42$ & 2.195 & $0.031^{*}$ & 0.279 \\
Psychosis & $1.91 \pm 0.76$ & $1.27 \pm 0.32$ & 4.996 & $0.000^{* *}$ & $0.000^{* *}$ \\
\hline
\end{tabular}

${ }^{* *} \mathrm{p}<0.01,{ }^{*} 0.01<\mathrm{p}<0.05$.

Table 2. Comparison of psychosocial factors between MS and healthy people

\begin{tabular}{lccrll}
\hline Item & MS group & Healthy group & $\mathrm{t}$ & $\mathrm{p}$ & $\mathrm{Pc}$ \\
\hline Life events scores & $39.37 \pm 40.75$ & $25.56 \pm 26.51$ & 1.818 & 0.073 & 0.073 \\
Positive life events scores & $5.80 \pm 10.46$ & $9.80 \pm 11.03$ & -1.685 & 0.096 & 0.192 \\
Negative life events scores & $34.99 \pm 37.21$ & $15.68 \pm 21.41$ & 2.790 & $0.007^{* *}$ & $0.014^{*}$ \\
Problems of family life & $29.98 \pm 31.91$ & $15.46 \pm 16.88$ & 2.574 & $0.012^{*}$ & $0.036^{*}$ \\
Problems of work and study & $6.59 \pm 11.98$ & $8.66 \pm 14.04$ & -0.719 & 0.474 & 1.422 \\
Social and other aspects & $4.27 \pm 9.23$ & $2.02 \pm 3.42$ & 1.460 & 0.148 & 0.444 \\
Utilization of social support & $6.95 \pm 1.80$ & $8.10 \pm 1.79$ & -2.893 & $0.005^{* *}$ & $0.015^{*}$ \\
Subjective social support & $24.34 \pm 4.65$ & $21.51 \pm 4.42$ & 2.825 & $0.006^{* *}$ & $0.018^{*}$ \\
Objective social support & $10.08 \pm 2.86$ & $10.68 \pm 2.59$ & -1.012 & 0.315 & 0.945 \\
P & $55.54 \pm 12.48$ & $52.20 \pm 7.75$ & 1.456 & 0.149 & 0.596 \\
E & $55.85 \pm 9.61$ & $59.27 \pm 10.22$ & -1.558 & 0.123 & 0.492 \\
$\mathrm{~N}$ & $54.39 \pm 10.56$ & $51.73 \pm 12.89$ & 1.022 & 0.310 & 1.240 \\
$\mathrm{~L}$ & $47.95 \pm 10.90$ & $47.93 \pm 9.29$ & 0.011 & 0.991 & 3.964 \\
\hline$\quad{ }^{* *} \mathrm{p}<0.01,{ }^{*} 0.01<\mathrm{p}<0.05$. & & & & & \\
\hline
\end{tabular}

er aspects, and in the four subscales of EPQ (table 2). These results suggested that the mental state in the healthy controls was better than in the MS patients.

\section{Relationship between Various Psychosocial Factors and Mental Health of MS Patients}

The scores for negative emotions in the MS group correlate positively with those of neurotic personality traits and negatively with those of introverted and extroverted traits, which suggests that emotional instability in patients with MS and introverted personality tend to cause negative emotion. A variety of negative emotions of the
MS group correlate positively with total number of life events, negative life events and family problems, suggesting that the occurrence of negative emotions was related to negative life events experienced during the 3 years before suffering from MS, and the impact brought about by family problems is particularly apparent. Many kinds of negative emotions correlated negatively with the utilization of social support in the MS group, suggesting that MS patients lacked the ability to use social support when they were under stress and lacked external resources that could reduce stress (table 3 ). 
Table 3. Relationship between various psychosocial factors and mental health of MS patients

\begin{tabular}{|c|c|c|c|c|c|c|c|c|c|}
\hline Item & Somatization & Obsession & $\begin{array}{l}\text { Interpersonal } \\
\text { relationship }\end{array}$ & Depression & Anxiety & Hostility & Horror & Paranoia & Psychosis \\
\hline Life events scores & $0.391^{* *}$ & $0.229 *$ & $0.271^{*}$ & $0.449^{* *}$ & $0.275^{*}$ & $0.243^{*}$ & 0.117 & 0.195 & $0.242^{*}$ \\
\hline Positive life events scores & 0.141 & 0.111 & 0.040 & -0.090 & 0.184 & -0.037 & 0.000 & 0.100 & -0.051 \\
\hline Negative life events scores & $0.409^{* *}$ & $0.229^{*}$ & $0.299^{* *}$ & $0.470^{* *}$ & $0.258^{*}$ & $0.278^{*}$ & 0.129 & 0.199 & $0.292^{* *}$ \\
\hline Problems of family life & $0.441^{* *}$ & $0.237^{*}$ & $0.326^{* *}$ & $0.437^{* *}$ & $0.254^{*}$ & $0.270^{*}$ & 0.165 & $0.228^{*}$ & $0.243^{*}$ \\
\hline Problems of work and study & 0.110 & 0.107 & -0.036 & 0.195 & 0.147 & -0.029 & 0.007 & -0.031 & 0.048 \\
\hline Social and other aspects & $0.222^{*}$ & 0.176 & 0.157 & $0.250^{*}$ & $0.247^{*}$ & -0.146 & -0.065 & $0.282^{*}$ & 0.194 \\
\hline Utilization of social support & $-0.238^{*}$ & -0.201 & $-0.294^{* *}$ & -0.207 & -0.174 & -0.090 & $-0.270^{*}$ & -0.182 & -0.194 \\
\hline Subjective support & 0.100 & -0.167 & -0.060 & -0.126 & -0.136 & $-0.242^{*}$ & 0.088 & -0.148 & -0.050 \\
\hline Objective social support & 0.030 & 0.017 & -0.089 & 0.003 & -0.022 & -0.210 & -0.098 & -0.164 & -0.101 \\
\hline $\mathrm{P}$ & 0.032 & -0.022 & 0.170 & 0.114 & 0.064 & 0.089 & 0.120 & $0.269^{*}$ & 0.178 \\
\hline $\mathrm{E}$ & -0.209 & $-0.337^{* *}$ & $-0.397^{* *}$ & $-0.353^{* *}$ & $-0.296^{* *}$ & -0.143 & $-0.277^{*}$ & -0.207 & $-0.393^{* *}$ \\
\hline $\mathrm{N}$ & $0.265^{*}$ & $0.421^{* *}$ & $0.354^{* *}$ & $0.431^{* *}$ & $0.380^{* *}$ & $0.299^{* *}$ & $0.231^{*}$ & $0.467^{* *}$ & $0.294^{* *}$ \\
\hline $\mathrm{L}$ & 0.024 & -0.032 & -0.036 & -0.039 & 0.001 & -0.121 & -0.121 & -0.138 & -0.071 \\
\hline
\end{tabular}

${ }^{* *} \mathrm{p}<0.01,{ }^{*} 0.01<\mathrm{p}<0.05$.

\section{Discussion}

The etiology of MS is thought to be an autoimmune process resulting from both hereditary susceptibility and environmental factors. Generally, the causes of MS can be categorized into two major types: hereditary and environmental [1]. The role of the hereditary cause has been confirmed by studies in many families and twins and it has been verified that MS susceptibility may be caused by the interaction of multiple genes. However, the hereditary factors can not fully explain the etiology of MS. Biological factors and psychosocial factors also play an important role in the etiology of MS. Over the years, a great number of papers on the etiology of MS have drawn more conclusions mainly from the biological aspect of MS than its psychosocial aspect. The psychosocial factors include changes in life, psychological conflicts and mental stress. In recent years, scholars have become more concerned with the psychological symptoms of MS patients. Researchers have found that $80 \%$ of MS patients show symptoms such as depression, sleep disorders, irritability or emotional instability and indifference [11]. Comparing relapse patients with those in remission, some studies have found that relapse MS patients showed symptoms of anxiety, negativeness, lack of self-confidence and avoidance of social activities [12]. Among the psychological symptoms, depression is of great concern, and it has a high correlation with the life quality of MS patients and their vocational ability [13-17]. As a result, other scholars have maintained that antidepressant treatment and psychological intervention could positively help patients cope with the disease [18-20]. All of these studies have been associated with the relationship between psychological factors and MS, and the overwhelming majority of authors believe that psychological disorders coexist with MS, or one of the MS symptoms. In addition, there is evidence to show that the relationship between MS relapse and life stress is complex and may rely on one or more factors such as long-term stress, severity, frequency and the type of the patient's personality and coping strategies [21, 22]. According to Folkman's stress model, characteristics of the stress source and personality of MS patients were only among the many intermediary factors affecting stress consequences. This is far from being sufficient to explain how the psychosocial factors affect the occurrence and development of MS, let alone establish the standards for screening high-risk groups and conducting further interventions based on the psychosocial factors.

In order to investigate and confirm the relationship between psychosocial factors and the onset of MS, we have conducted a retrospective examination of the patients with MS by the LES, EPQ, SCL-90 and SSRS questionnaires, which are classical and culturally adapted. These psychological tests have been well established in China for many years and the standardized psychological testing and multivariate analysis methods were used to examine the functions of psychosocial stress, intermediary factors and their interaction. Our data indicate that some important or sustained negative life events may be related to the pathogenesis of MS. We also identified the related risk factors to establish a theory and intervention approach to cope with stress. 
Significant differences between the MS and the control group were found in their negative emotions and symptoms such as depression, anxiety, obsession, phobia, tense interpersonal relationship and somatization disorder (table 1). Furthermore, significant differences in total number of negative life events, family problems and utilization of social support were found between the two groups. There were no significant differences in the four subscales of EPQ (table 2). These results suggest that the mental state is better in the healthy controls than in MS patients. The scores for negative emotions in the MS group correlated positively with those for neuroticisms in personality type, and negatively with those for introverted and extroverted personality traits (table 3), suggesting that the emotional instability and introverted personality of patients with MS led to the occurrence of negative emotions. A variety of negative emotions in the MS group correlated positively with total number of life events, negative life events and family problems (table 3), suggesting that the occurrence of negative emotions was related to negative life events experienced 3 years before they were diagnosed as MS, especially the impact family problems brought about. Many kinds of negative emotions in the MS group correlated negatively with the utilization of social support (table 3), suggesting that the patients lacked the ability to use social support which can usually reduce it. As a result, the risk of incidence could increase when an individual is under stress without social support. Our data clearly indicate a link between the psychosocial factors and the pathogenesis of MS.

According to the above data, we speculate that the perprotocol susceptible population, which lacks social sup- port or has low utilization of social support, suffered intense psychological stress, which resulted in immune responses [4] that might lead to MS. Further study should be carried out to obtain the data about immune responses.

With the establishment of the biopsychosocial medical model, the simpler biomedical model cannot provide satisfactory explanation for human diseases when the psychosocial factors exert an impact on health, and illness has been a widespread concern. Psychosocial factors are closely linked to the pathogenesis, pathophysiology and clinical symptoms of MS. The important role they play in the onset, recurrence and severity of the disease cannot be ignored. The present study has shown a very interesting field to explore the etiology of MS by the biopsychosocial medical model. It may give a more comprehensive, scientific and complete explanation for the pathogenesis of MS. Therefore, we can implement comprehensive multi-channel control of the causes of MS from the physiological, psychological and social perspective to reduce MS incidence and improve the life quality of patients.

\section{Acknowledgements}

We wish to thank the doctors from the Department of Neurology of Nanfang Hospital in Guangdong Province, China, for their preparation of medical records of some participants and neuropsychological tools, and thank Prof. Cheng Ping-yan from the Department of Statistics at the Southern Medical University for statistical support. We would also like to thank the patients who kindly participated in this study.

\section{References}

1 Rumrill PD, Kaleta DA, Battersby JC: Etiology, incidence, and prevalence; in Rumrill PD, (ed): Employment Issues and Multiple Sclerosis. New York, Demos Vermande, 1996, pp 1-18.

-2 Brassington JC, Marsh NV: Neuropsychological aspects of multiple sclerosis. Neuropsychol Rev 1998;8:43-77.

-3 Osborne TL, Jensen MP, Ehde DM, et al: Psycho-social factors associated with pain intensity, pain-related interference, and psychological functioning in persons with multiple sclerosis and pain. Pain 2007;127: $52-62$.
4 Strenge H: The relationship between psychological stress and the clinical course of multiple sclerosis. An update. Psychother Psychosom Med Psychol 2001;51:166-175.

5 Brown RF, Tennant CC, Sharrock M, et al: Relationship between stress and relapse in multiple sclerosis. II. Direct and indirect relationships. Mult Scler 2006;12:465-475.

6 Beatty WW, Paul RH, Wilbanks SL, et al Identifying multiple sclerosis patients with mild or global cognitive impairment using the Screening Examination for Cognitive Impairment. Neurology 1995;45:718-723.

7 Yang D, Zhang Y: Life Event Scale. Mental Health Assessment Scale Manual. Chin Ment Health J 1993;39-41.
8 Gong Y: Eysenck Personality Questionnaire Manual. Changsha, Hunan Medical College, 1987, pp 31-44

9 Zhang M: Behavioral medicine Scale Manual. Chin Behav Med Sci 2001;1182-1211.

10 Xiao S, Yang D: Social Support Revaluate Scale. Mental Health Assessment Scale Manual. Chin Ment Health J 1993;44-45.

11 Figved N, Klevan G, Myhr KM, et al: Neuropsychiatric symptoms in patients with multiple sclerosis. Acta Psychiatr Scand 2005; 112:463-468.

12 Papuc E, Pawlowska B: Personality features in multiple sclerosis patients with a relapsing-remitting course of the disease. Psychiatr Pol 2005;39:669-678. 
13 Goldman Consensus Group: The Goldman Consensus statement on depression in multiple sclerosis. Mult Scler 2005;11:328-337.

14 Ehde DM, Bombardier CH: Depression in persons with multiple sclerosis. Phys Med Rehabil Clin N Am 2005; 16:437-448.

15 Zorzon M, Masi R, Nasuelli D, et al: Depression and anxiety in multiple sclerosis. A clinical and MRI study in 95 subjects. J Neurol 2001;248:416-421.

16 Chen L, Wu W, Mao Y, et al: Nine cases of multiple sclerosis characteristics of depression, anxiety disorders and related factors. Chin J Pract Intern Med 2004;24:239-242.
17 Buchanan RJ, Zhu L, Schiffer R, et al: Ruralurban analyses of health-related quality of life among people with multiple sclerosis. J Rural Health 2008;24:244-245.

18 Ye J: Effect of paroxetine on multiple sclerosis with depression and neurological functional rehabilitation. Chin J Rehab Theory Pract 2005;11:131-132.

19 Hart S, Fonareva I, Merluzzi N, et al: Treatment for depression and its relationship to improvement in quality of life and psychological well-being in multiple sclerosis patients. Qual Life Res 2005;14:695-703.
20 Patten SB: Treatment of neuropsychiatric syndromes in multiple sclerosis. Expert Rev Neurother 2005;5:413-420.

21 Chalk HM: Mind over matter: cognitivebehavioral determinants of emotional distress in multiple sclerosis patients. Psychol Health Med 2007;12:556-566.

22 Brown RF, Tennant CC, Dunn SM, et al: A review of stress-relapse interactions in multiple sclerosis: important features and stressmediating and -moderating variables. Mult Scler 2005;11:477-484. 